\title{
LA INFLUENCIA DE LOS FACTORES TAFONÓMICOS Y PALEOECOLÓGICOS EN LA DISTRIBUCIÓN DE LOS MOLUSCOS EN EL ÁREA TIPO DEL ILERDIENSE (CONCA DE TREMP, CATALUÑN, ESPAÑA)
}

\author{
Miquel DE RENZI \\ ' Departament de Geología. Universitat de València. Campus de Burjassot. Burjassot 46100 Valencia.
}

\begin{abstract}
De Renzi, M. 1996. La influencia de los factores tafonómicos y paleoecológicos en la distribución de los moluscos en el área tipo del Ilerdiense (Conca de Tremp, Cataluña, España). [The influence of taphonomic and paleoecologic factors in the distribution of moluscs in the type area of the Ilerdian (Conca de Tremp, Catalonia, Spain)]. Revista Española de Paleontología, $\mathbf{N}^{*}$ Extraordinario, 204-214. ISSN 0213-6937.
\end{abstract}

\begin{abstract}
Now, the Ilerdian has no world-wide value, although it is a well admitted stage in a regional scale (Tethys). This paper deals with the stratigraphical value of molluscs in the Ilerdian type area. Their distribution is controlled by both taphonomical and paleoecological factors in a transgressive-regressive megasequence. The earliest occurrence of many molluscan species is very influenced by environmental conditions, with the exception of those from Turritella marls, whereas preservation of aragonitic forms is only frequent in a part of the type area. Several species occurring in the Ilerdian type area for first time appear later in other important basins. However, they are good markers for the type area of the Ilerdian.
\end{abstract}

Keywords: Ilerdian, Molluscs, Stratotypes, Taphonomy, Paleoecology, Biostratigraphy.

\section{RESUMEN}

\begin{abstract}
Aunque no se le reconozca actualmente un valor mundial, el llerdiense es un piso bien admitido a escala regional (Tethys). Este trabajo trata del valor estratigráfico de los moluscos en su área tipo. Su distribución está controlada por factores tafonómicos y paleoecológicos en una megasecuencia transgresivo-regresiva. Sus primeras apariciones están muy influidas por las condiciones ambientales, excepto los de las margas de Turritella, mientras que la conservación de las formas aragoníticas es sólo frecuente en una parte de la Conca de Tremp. Varias especies que aparecen en el área tipo del Ilerdiense por vez primera, no lo hacen en otras cuencas importantes hasta más tarde. Sin embargo, resultan buenas marcadoras para el área tipo del Ilerdiense.
\end{abstract}

Palabras clave: Ilerdiense, Moluscos, Estratotipos, Tafonomía, Paleoecología, Bioestratigrafía.

\section{INTRODUCCIÓN}

El Ilerdiense fue un piso definido en 1960 para resolver algunos de los problemas que planteaba la división clásica en pisos del Paleoceno y del Eoceno a partir de las sucesiones de la cuenca de París (Hottinger y Schaub, 1960). Tales problemas hacían referencia a que en las cuencas meridionales (España, Sur de Francia, Italia, etc.) existirían sucesiones muy continuas que sólo podían correlacionarse en parte con la escala de la cuenca de París, mientras que determinadas porciones de las mismas no tendrían equivalente en aquélla.

Hottinger y Schaub designaron como localidad tipo del Ilerdiense la Conca de Tremp, en la provincia de Lérida, en los materiales marinos de edad paleógena. Estos materiales fueron estudiados por vez primera por Carez (1881). Para Carez, serían de la misma edad que los de la región francesa de Les Corbières (Aude, Francia), que Leymerie acababa de estudiar; también serían contemporáneos de los sables de Cuise, en la cuenca de París, y de los sedimentos de Spilecco y Monte Postale (Italia). La conclusión fue que estos materiales serían atribuibles al Eoceno inferior; sin embargo, Vidal posteriormente hacía ya vagas asignaciones a "Nummulítico inferior" y "Nummulítico medio" en sus envíos de moluscos a Cossmann, que este último mantuvo (Cossmann, 1897).

La correlación entre los terrenos marinos de la Conca de Tremp y de Les Corbières hizo que la problemática de su edad corriera suertes paralelas. Doncieux (1903, 1905, 1908, 1911) da una visión completamente distinta a la de Leymerie. Para él, el Eoceno inferior (Thanetiense, Esparnaciense y Cuisiense) únicamente constituiría la base de los materiales marinos de aquella región francesa, mientras que las secuencias margosas suprayacentes con Turritella tendrían edad luteciense.

Dalloni (1930), en su estudio sobre los Pirineos catalanes, adopta las ideas de Doncieux, ya que la sucesión de litologías y fósiles es, a grandes rasgos, parecida. Esta datación permaneció prácticamente inmodificada hasta los estudios de Hottinger y Schaub (1960), que condujeron a la definición del nuevo piso. De acuerdo con la misma, se volvía a colocar dichas capas en una posición baja dentro del Paleógeno. El piso propuesto vendría representado por los cuerpos rocosos depositados entre el Montiense superior y el Cuisiense, sin 
correlato en la cuenca de París. Aunque el Paleoceno representara -y sigue representando- un problema en lo referente a sus límites, el Ilerdiense fue asimilado a un "Paleoceno con nummulites", ya que quedaría por debajo del límite inferior del Ypresiense, tanto en la Conca de Tremp como en el Schlierenflysch alpino. Secuencias de ese tipo no se conocían en la cuenca de París, con lo cual la correlación era imposible y, de aquí, la justificación del nuevo piso. Ello afectaría también a los terrenos homónimos de Les Corbières (Hottinger, 1960; Plaziat y De Renzi, 1968). En De Renzi $(1966,1968)$ se dieron dataciones para los materiales infrayacentes al Ilerdiense (Montiense-Thanetiense), intercalados en la facies garumniense de la sección del Isábena, y para los suprayacentes (Cuisiense marino) en la misma sección del Isábena y al S de Puente de Montañana.

Posteriormente se ha visto (cf. Molina et al., 1992) que el Ilerdiense medio y el superior se solapan con el Ypresiense. Únicamente el Ilerdiense inferior constituiría, de acuerdo con dichos autores, el registro estratigráfico y paleontológico (boundary time span) de una laguna presente entre el Thanetiense y el Ypresiense, aunque sin entidad suficiente, por su brevedad, para mantenerse como piso estándar en la escala de tiempo geológico. No obstante lo dicho, sigue siendo muy útil como piso regional para las plataformas del Tethys (Molina, comunicación personal).

La propuesta del nuevo piso hizo que se estudiaran sus contenidos paleontológicos (por ejemplo, Luterbacher, 1970). Uno de estos estudios fue el de los Moluscos, que constituyó el objeto de mi tesis doctoral inédita (De Renzi, 1971), aunque consultada por diversos autores y citada en diversos trabajos (cf. Luterbacher, 1973; Llompart, 1977; Barnolas et al. 1990). La elaboración de la misma dio origen a diversos trabajos previos directa o indirectamente relacionados con el tema (De Renzi, 1966, 1967, 1968, 1972, 1975; Crusafont et al. 1968; Crusafont, Rosell et al. 1968; Plaziat y De Renzi, 1968). Sus precedentes fueron los trabajos de Cossmann $(1897,1906)$, así como también los listados de Dalloni (1930) que, sin embargo, no contenían ni descripciones ni ilustraciones.

La presente síntesis va a tratar de la repartición horizontal y vertical de los Moluscos, así como también de sus controles, junto con las conclusiones revisadas de De Renzi (1971), tanto por aporte de nuevos datos como de nuevas ideas.

\section{LOS MOLUSCOS MARINOS DEL ILERDIENSE DE TREMP}

Los moluscos del Paleógeno marino de la Conca de Tremp constituyen un material diversificado en la zona de estudio, aunque desigualmente repartido. Se cuentan 108 especies, de las cuales 82 son de gasterópodos y 26, de bivalvos. Los gasterópodos se distribuyen entre 46 géneros, 21 familias y 3 órdenes. Las especies de bivalvos están repartidas entre 21 géneros, 12 familias y 7 órdenes. Las tablas 1 y 2 muestran su listado y distribución estratigráfica; esto último se comentará más adelante.

\section{Secciones estratigráficas}

Desde un punto de vista litoestratigráfico, los niveles marinos que constituyen la sección tipo y el área tipo del Iler-

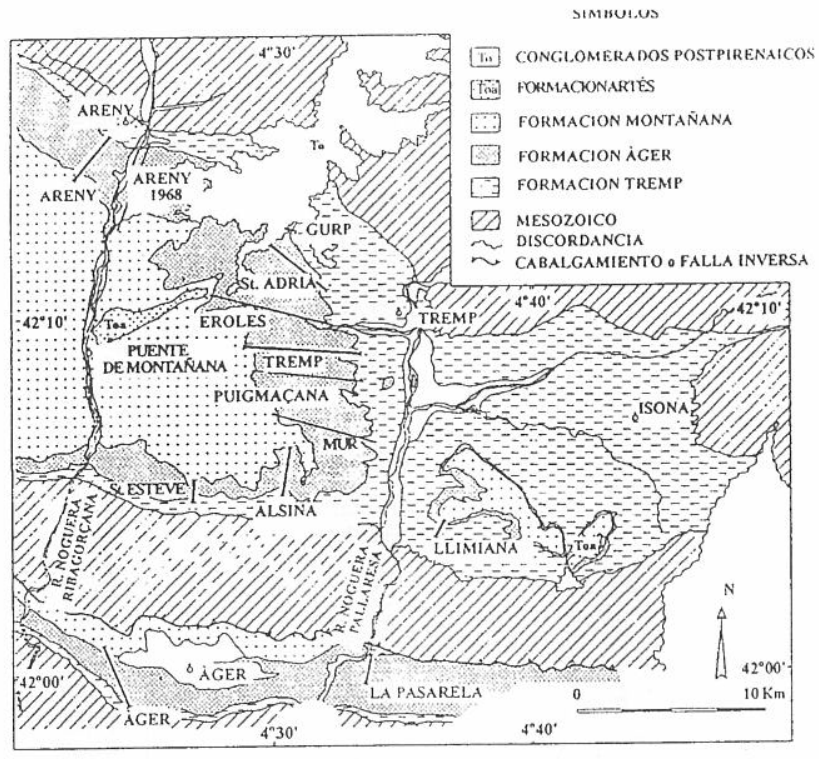

Figura 1. Mapa de Ferrer et al. (1973) donde se ubican la secciones levantadas por mi (ver texto): Mur-Collmorté, Puigmaçana-Puigvert, Tremp-Puente de Montañana, Tendruy-Sant Adrià y Gurp (De Renzi, 1967); Areny (1968) (De Renzi, 1968).

diense, pertenecen a la Formación Ager - una megasecuencia transgresivo-regresiva-, que se sitúa, a su vez, por encima de la Formación Tremp y por debajo de la Formación Montañana, ambas continentales (fide Ferrer et al. 1973), aunque esta nomenclatura ha variado, se ha modificado y, posiblemente, habrá nuevos cambios (Serra-Kiel et al. 1994). Hacia el N se disponen los materiales del Eoceno superior-Oligoceno, en contacto erosivo con las capas de estas tres formaciones.

La localización de los moluscos fósiles llevó a levantar diversas secciones estratigráficas (Fig. 1), algunas de ellas coincidentes en parte con las ya levantadas por Hottinger (1960): la de Mur, la de Puigmaçana y la de la carretera de Tremp a Puente de Montañana. Las otras no levantadas hasta entonces (De Renzi, 1967) fueron: una por Eroles, otra pasando por Tendruy y Sant Adrià, y una última al S de Gurp (muy cerca del campamento militar, que todavía subsiste como escuela de suboficiales). Estas localizaciones fueron visitadas posteriormente por otros autores (Luterbacher, 1970 -corte por Tendruy y Sant Adrià-; el mismo por Ferrer et al. 1973; en ambos casos, los objetivos se referían a micropaleontología; concretamente, foraminíferos). Al año siguiente (De Renzi, 1968), se publicaron tres nuevos cortes: uno, al E de Areny; otro, por Serraduy, Puebla de Roda y Roda de Isábena, y un último al S de Puente de Montañana. De estas tres últimas secciones, únicamente entraron en las descripciones sistemáticas los moluscos del este de Areny. Para los moluscos de las facies más altas de La Vall d'Ager me basé en la columna estratigráfica publicada en Crusafont, Rosell et al. (1968).

Luterbacher (1970) señala cortes por Sant Esteve, por Alsina, por Llimiana y por La Pasarela (al S del Montsec), que ya no visité (la memoria de tesis estaba prácticamente finalizada y la información era parecida, aunque pudiera haber algunas novedades puntuales). Dichos cortes son estudiados también por Ferrer et al. (1973). 


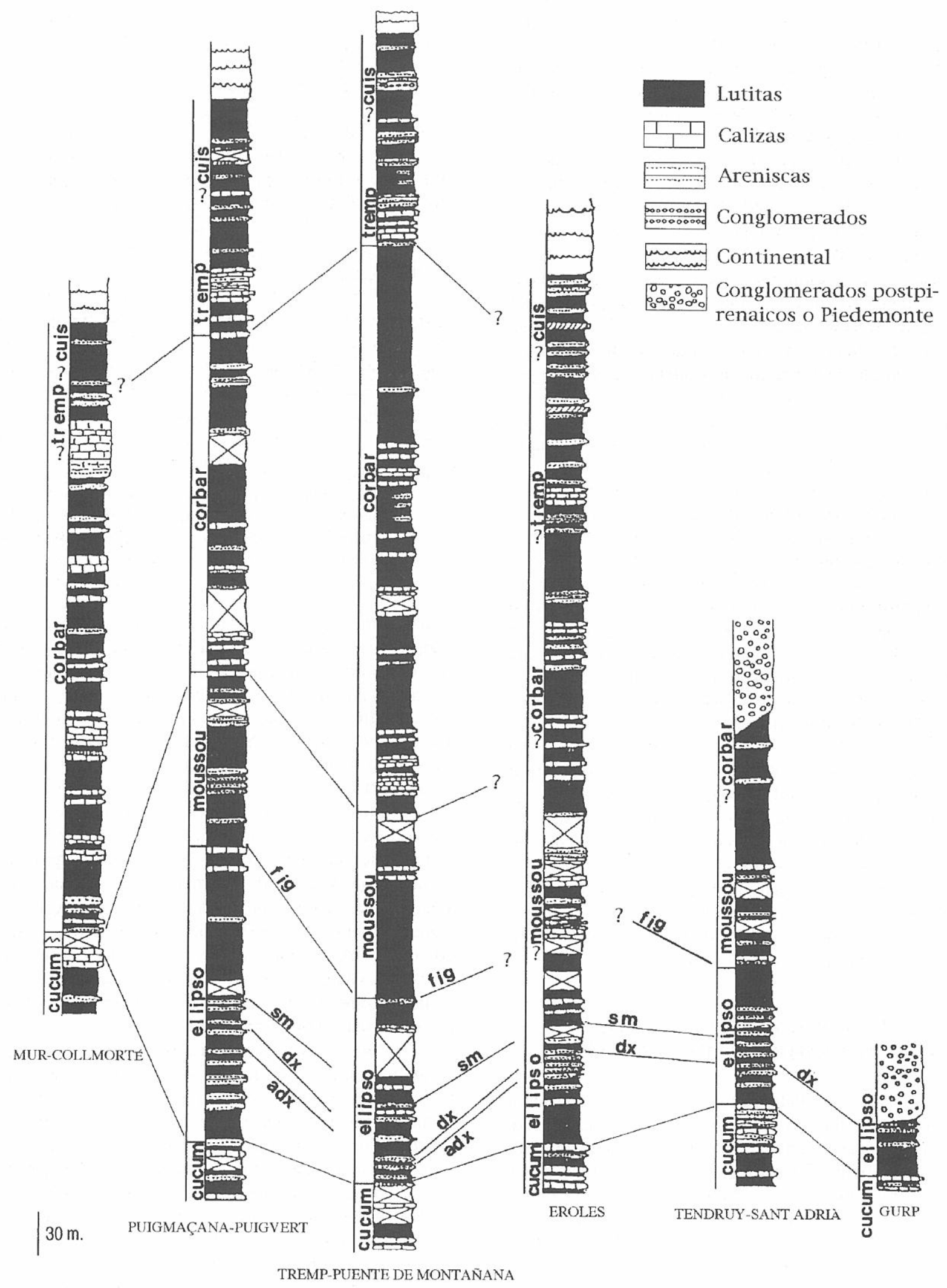

Figura 2. Secciones por Mur-Collmorté, Puigmaçana-Puigvert, Tremp-Puente de Montañana, Eroles, Tendruy-Sant Adrià y Gurp (de los datos de De Renzi, 1971, aunque no dibujadas allí). Leyenda: cucum, Biozona Cucumiformis; ellipso, Biozona Ellipsoidalis; moussou, Biozona Moussoulensis; corbar, Biozona Corbarica; tremp, Biozona Trempina, y cuis, Cuisiense inferior. fig, primera aparición de Turritella figolina; sm, primera aparición de Sigmesalia spp.; dx, primera aparición de Turritella dixoni; adx, primera aparición de Turritella spp. aff. dixoni. 
Las columnas muestran (Fig. 2) como al S de la Conca de Tremp -y todavía más en cortes no representados aquí, como en Alsina o Sant Esteve- los materiales calcáreos y detríticos más o menos groseros se disponen en bancos potentes, mientras que a medida que avanzamos hacia el $\mathrm{N}$, las secciones muestran una mayor proporción de lutitas (margas grises), que alternan con hiladas detríticas organógenas. Hay que decir que el corte por Eroles representaría la sección más al $\mathrm{N}$ de las facies marinas no cubierta por los conglomerados o el piedemonte. El corte por Areny representaría una continuación de las facies lutíticas del centro y $\mathrm{N}$ de la Conca, mientras que el levantado por el S de Puente de Montañana (ver, para ambos, De Renzi 1968) estaría en conexión con el predominio calcáreo-detrítico observado al S de Tremp (Mur, Alsina, Sant Esteve). Estas observaciones nos serán útiles a la hora de hablar de la tafonomía y paleoecología de las asociaciones de moluscos de la zona objeto de estudio.

\section{Tafonomía}

Los gasterópodos tienen conchas constituidas por aragonito, o bien biminerálicas (aragonito+calcita de bajo contenido en $\mathrm{Mg}$ ), mientras que los bivalvos, aparte de estos dos tipos mineralógicos, pueden construir conchas monominerálicas de calcita con bajo contenido en $\mathrm{Mg}$. En ambos casos, las conchas biminerálicas o las monominerálicas de calcita son las más conservables en la fase fosildiagenética. El aragonito suele desaparecer, en esta fase, o ser reemplazado por algún tipo de cemento; al disolverse, las conchas aragoníticas dejan únicamente moldes, y si ello ocurre durante la fase bioestratinómica, no dejan rastro.

Los materiales calcáreos y detríticos más o menos groseros de la Conca de Tremp contienen conchas fósiles de moluscos calcíticos o biminerálicos; estas son de géneros tales como Ostrea (s.l.), Spondylus, etc. todos ellos bivalvos. Los fósiles de especies aragoníticas se hallan, a lo más, en forma de moldes. Los moldes internos de bivalvos y gasterópodos terciarios suelen ser, por regla general, prácticamente indeterminables.

Los materiales lutíticos más arcillosos sí que contienen conchas inicialmente aragoníticas, pero siempre neomorfizadas en calcita, en estados de conservación desiguales y, en algunos casos, deformadas. Sus superficies suelen presentar, bien conservados, las líneas de crecimiento y diversos aspectos delicados. Los bivalvos de las lutitas están articulados y cerrados -por lo menos en esta zona-, lo cual parece indicar enterramientos relativamente rápidos y/o fondos muy tranquilos, dado que muchos de los bivalvos son endobiontes (ver Tabla 3). Precisamente, el carácter cerrado de estas conchas fue un verdadero obstáculo para el estudio sistemático, ya que el conocimiento de la charnela es normalmente esencial para la determinación.

Mención aparte merecen las concentraciones -elementos acumulados o resedimentados; ver más abajo- de conchas de Turritella en lutitas. Se hace difícil ver otros fósiles de moluscos aragoníticos junto a ellas (hay algún caso muy particular en la sección de la carretera de Tremp a Puente de Montañana, con micromoluscos); por lo general, si aparecen suelen estar en muy mal estado. Ello plantea la cuestión de si las Turritella no podrían poseer una característica de su mineralogía o de su microestructura que favoreciera su conservación diferencial (Seilacher, comunicación personal).

A pesar de lo dicho, los materiales margosos situados por encima de las margas con Turritella no contienen generalmente fósiles de conchas aragoníticas en su origen. Si lo hacen, el estado de los mismos es bastante deficiente por lo general: fragmentos muy alterados de concha adheridos a moldes internos, o bien éstos desnudos. Es probable que ello se deba a la mayor permeabilidad, dado que se trata de lutitas limosas. No obstante ésto, la especie típica de las margas de Turritella ( $T$. dixoni Deshayes) es reconocible más de una vez en dichas capas. Ello nos indicaría dos tafofacies distintas.

Por último, los depósitos margosos en la parte más alta de la formación marina vuelven a contener fósiles de moluscos aragoníticos neomorfizados en calcita, cuya conservación es tanto mejor cuanto más al $\mathrm{N}$ de la Conca vayamos.

Los fósiles de los materiales detríticos más o menos groseros, incluidos los moluscos, suelen presentar carácter resedimentado. En cuanto a las margas con Turritella, también se observan orientaciones debidas a corrientes locales en las conchas de estas últimas, típicamente alargadas. En otros casos, la dispersión de Turritella en el sedimento parece concorde con una energía muy baja, y los ejemplares suelen estar muy completos; los corales solitarios asociados a ellas acostumbran también a estar muy enteros y bien conservados. Los ostreidos, otro grupo importante en el Ilerdiense tipo, están por lo general desarticulados, a excepción de algunas bioconstrucciones visibles tanto hacia la base (corte de Eroles) como hacia el techo de las secciones. Los fenómenos de bioerosión e incrustación -perforaciones, corales incrustantes, etc.- se hacen patentes con frecuencia en los ostreidos. Aunque ello no es tan frecuente, las Turritella también presentan ese tipo de señales.

\section{Paleoecología}

Si bien en De Renzi (1971) -también De Renzi (1975)hay un intento de aproximación paleoautoecológica, de un uniformismo estricto reconocido explícitamente desde buen principio -esta misma aproximación sigue Llompart (1977)-, sólo el trabajo de Luterbacher (1970) da las bases para repartir los moluscos en unos ambientes concretos; otros trabajos se han seguido posteriormente, como el ya clásico de Mutti et al. (1972) o el muy reciente, que recoge otros, de Serra-Kiel et al. (1994). La aproximación uniformista sustantiva padece de defectos graves (cf. De Renzi, 1978, 1981, 1988a) y en esta síntesis se va a optar más bien por una via concorde con un uniformismo metodológico, basado en interpretaciones del significado del tamaño y de los rasgos morfológicos, así como también de aspectos tales como densidad de fósiles; es obvio que todo esto tendrá sentido únicamente tras un análisis tafonómico previo, y con todas las salvedades que pueda haber en cuanto a interpretación funcional de los diversos aspectos morfológicos de los moluscos aquí estudiados.

Las facies basales corresponden a ambientes muy someros (medios parálicos) en que las influencias marinas se hacen sentir. Estas últimas estarían marcadas por la presencia de alveolínidos y otros grandes foraminíferos netamente marinos (Hottinger, 1984), pero también por moluscos muy característicos; así, la presencia de grandes Pseudomiltha (?) 


\begin{tabular}{|c|c|c|c|c|c|c|}
\hline \multirow{2}{*}{$\begin{array}{l}\text { GASTEROPODOS-ESPECIES } \\
\end{array}$} & \multicolumn{6}{|c|}{ DISTRIBUCION ESTRATIGRAFICA } \\
\hline & cucu & clli & mouss & corbar & tremp & cuis \\
\hline \multirow{8}{*}{$\begin{array}{l}\text { Tectus (s.s.) pyrenaicus Villatte } \\
\text { Angaria doncieuxi Villatte } \\
\text { Liotina decipiens } \text { (Bayan) } \\
\text { Homalopoma (s.s.) conoideum (Doncieux) } \\
\text { Otostoma sp. } \\
\text { - vidali Cossmann } \\
\text { Sigmesalia spp. }\end{array}$} & & E,A & & & & \\
\hline & $E$ & E,T,A & ?T??A & & & \\
\hline & & & & & & $\mathrm{AG}$ \\
\hline & G & $\mathrm{E}, \mathrm{A}$ & & & & \\
\hline & G & $\mathrm{E}, \mathrm{A}$ & & & & \\
\hline & & & & & & $\mathrm{AG}$ \\
\hline & & $\begin{array}{c}\mathrm{P}, \mathrm{PM}, \mathrm{E}, \mathrm{T} \\
\mathrm{G}, \mathrm{A}\end{array}$ & $? \mathrm{~T}$ & & & \\
\hline & & & & & & $\begin{array}{l}\mathrm{M}, \mathrm{P} \\
\mathrm{AG}\end{array}$ \\
\hline \multirow{3}{*}{$\begin{array}{l}\text { Zaria (?) vaudini (Deshayes) } \\
\text { - (?) quinquecarinata (Doncieux) } \\
\text { - (?) caroli Doncieux }\end{array}$} & PM & $T$ & & & & \\
\hline & PM & E,A & & & & \\
\hline & & $\mathrm{T}$ & $? \mathrm{~T}$ & $\mathrm{P}$ & & \\
\hline \multirow{3}{*}{$\begin{array}{l}\text { Turritella funiculosa (Deshayes) } \\
\text { - aff. carinifera Deshayes } \\
\text { - dixoni Deshayes }\end{array}$} & PM & $\ldots+\cdots$ & $\cdots \cdots$ & $\ldots+\ldots$ & $\ldots$ & PM \\
\hline & & $T$ & ?T & $\ldots$ & $\ldots$ & $\mathrm{AG}$ \\
\hline & & $\begin{array}{c}\mathrm{P}, \mathrm{PM}, \mathrm{E}, \mathrm{T} \\
\mathrm{G}, \mathrm{A}\end{array}$ & T & $\mathrm{T}$ & $\cdots$ & $E$ \\
\hline \multirow{5}{*}{$\begin{array}{l}\text { - spp. aff. dixoni Deshayes } \\
\text { - figolina Carez } \\
\text { - peraubensis Cossmann } \\
\text { - dufrenoyi Leymerie }\end{array}$} & & $\begin{array}{c}\mathrm{P}, \mathrm{PM}, \mathrm{E}, \mathrm{T} \\
\mathrm{A}\end{array}$ & & & & \\
\hline & & & P.PM,T,A & & & \\
\hline & & & & & & $\mathrm{AG}$ \\
\hline & & \begin{tabular}{|c|} 
PM,E,G \\
A
\end{tabular} & $\begin{array}{c}\text { PM } \\
? \mathrm{~T}, ? \mathrm{~A} \\
\end{array}$ & & & \\
\hline & & PM & & & & \\
\hline \multirow{2}{*}{$\begin{array}{l}\text { Melanatria almerae (Vidal in Cossmann) } \\
\text { - vulcanica (Schlothcim) }\end{array}$} & P,PM,E & $\ldots$ & $\ldots$ & $\ldots+\cdots$ & $\ldots$ & $\mathrm{E}$ \\
\hline & & & & & & $\mathrm{AG}$ \\
\hline \multirow{2}{*}{$\begin{array}{c}\text { Melanopsis (s.s.) ancillaroides Deshayes } \\
\text { - (Canthidomus) vidali (Cossmann) }\end{array}$} & & & & & & $\mathrm{AG}$ \\
\hline & PM,E,T & & & & & \\
\hline \multirow{6}{*}{$\begin{array}{l}\text { Potamides (Ptychopotamides) aff. cinctus } \\
\quad \text { (Bruguière) } \\
\text { - - cf. solerensis (Carez in Cossmann) } \\
\text { - (Alocaxis) aff. cylindraceus } \\
\text { (Deshayes) } \\
\text { - (Potamidopsis) imbricatarius Cossmann } \\
\text { - peraubensis Cossmann } \\
\text { - (Exechestoma) cf. suturatus Cossmann }\end{array}$} & E & $\ldots$ & & -......-- & $\ldots$ & TRP \\
\hline & & & & & & $\mathrm{AG}$ \\
\hline & $\mathrm{PC}$ & & & & & \\
\hline & PM & & & & & \\
\hline & & & & & & $\mathrm{AG}$ \\
\hline & & & & & & $\begin{array}{l}\mathrm{M}, \mathrm{P} \\
\mathrm{ME}\end{array}$ \\
\hline \multirow[t]{2}{*}{ Pirenella (Tiaracerithium) almerae (Cossmann) } & $\begin{array}{l}\mathrm{P}, \mathrm{PC}, \mathrm{E} \\
\mathrm{PM}, \mathrm{T} \\
\end{array}$ & & & & & \\
\hline & & & & & & $\mathrm{AG}$ \\
\hline \multirow{3}{*}{$\begin{array}{l}\text { Tympanotonos (Eotympanotomus) hypermeces } \\
\text { (Cossmann) } \\
\text { - - inaequirugatus (Cossmann) } \\
\text {. - turris orengae (Vidal in Cossmann) }\end{array}$} & $\begin{array}{c}\mathrm{P}, \mathrm{PM}, \mathrm{PC} \\
\mathrm{E}, \mathrm{T}\end{array}$ & & & & & \\
\hline & PM,E.T & & & $\cdots+\cdots$ & $\ldots$ & $\mathrm{AG}$ \\
\hline & $\begin{array}{c}\mathrm{P}, \mathrm{PC}, \mathrm{PM} \\
\mathrm{E}\end{array}$ & & -............ & $? \mathrm{P}$ & ..... & \begin{tabular}{|l|}
$\mathrm{M}$ \\
$\mathrm{AG}$ \\
\end{tabular} \\
\hline \multirow{2}{*}{$\begin{array}{l}\text { - (Diptychochilus) montsecamus (Vidal in } \\
\text { Cossmann) } \\
\text { Cerithidea vidali (Cossmann) }\end{array}$} & & & & & & $\begin{array}{c}M . P M \\
A G\end{array}$ \\
\hline & PM & & & & & \\
\hline Tylochilus palensis (Rouault) & & & & & & $\mathrm{AG}$ \\
\hline - tactospira (Cossmann) & & & & & & $\mathrm{AG}$ \\
\hline \multirow{2}{*}{$\begin{array}{l}-(?) \text { vidali (Cossmann) } \\
- \text { multigranosus (Doncicux) }\end{array}$} & & & & & & AG \\
\hline & & & & & & $\mathrm{M}, \mathrm{P}$ \\
\hline
\end{tabular}

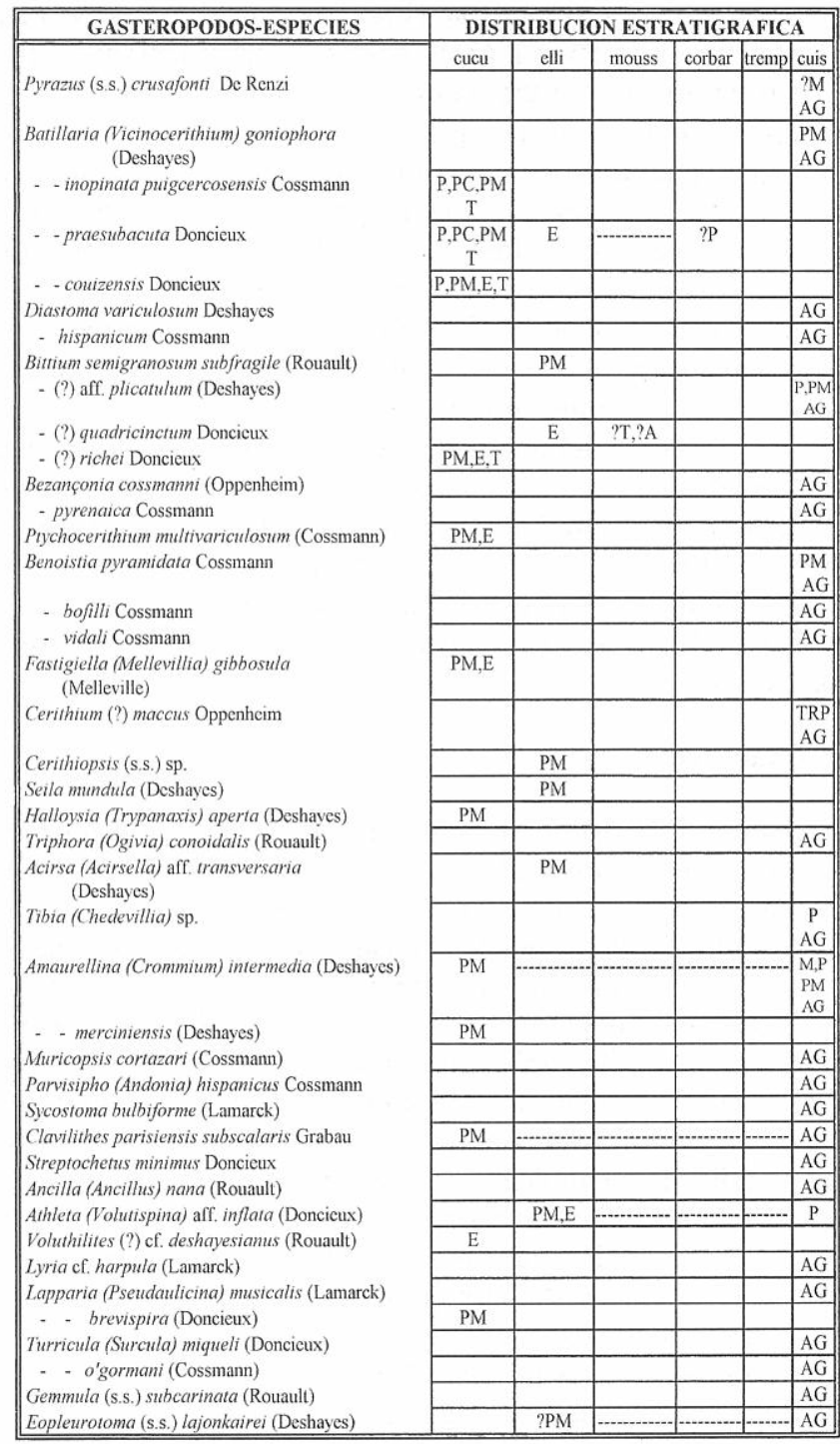

Tabla 1. Listado de las especies de gasterópodos marinos de la Conca de Tremp y La Vall d'Ager, con su distribución estratigráfica y por yacimientos, según los datos y orden taxonómico de De Renzi (1971). Siglas (refieren a los cortes): M, Mur. P, Puigmaçana. PM, Puente de Montañana. PC, Puigcercós. E, Eroles. T, Tendruy-Sant Adrià. A, Areny. AG, Àger. TRP, Tremp (indiscriminado); cucu, Biozona Cucumiformis; elli, Biozona Ellipsoidalis; mouss, Biozona Moussoulensis; corbar, Biozona Corbarica; tremp, Biozona Trempina; cuis, Cuisiense inferior.

corbarica (Leymerie) indicaría de alguna manera esta influencia marina, en zonas de relativa energía (mareas, etc.). Los Lucinacea acostumbran a enterrarse más o menos profundamente; las grandes Pseudomiltha suelen presentarse en posición de producción en sedimentos arenosos bastante clasificados, aunque también son frecuentes en lutitas (zonas de lagoon; Luterbacher, 1970). Su gran tamaño, el grosor de sus conchas (uno de los mayores moluscos de esta zona) y la densidad relativamente baja en el sedimento hacen pensar en un medio francamente marino y estable en cuanto a condiciones generales $\left(\mathrm{O}_{2}\right.$, salinidad, etc.), propio para el establecimiento de los estrategas de $K$. Otros moluscos típicamente marinos son los de la Familia Cerithiidae; aparecen como elementos resedimentados en niveles con alveolínidos resedimentados a su vez. Podemos mencionar Ptychocerithium multivariculosum (Cossmann) y Fastigiella (Mellevillia) gibbosula (Melleville).
En cambio, los ambientes parálicos estarían poblados por moluscos salobres muy típicos: los de la Familia Potamididae (cf. Fischer, 1887; aunque ver también Plaziat, 1989, 1993, 1995; según este último autor, también podrían colonizar litorales marinos protegidos y lagos salados litorales). Así, Potamides (Ptychopotamides) aff. cinctus (Bruguière), Potamides (Potamidopsis) imbricatarius Cossmann, Pirenella (Tiaracerithium) almerae (Cossmann), Tympanotonos (Eotympanotonus) hypermeces (Cossmann), Batillaria (Vicinocerithium) inopinata puigcercosensis Cossmann, etc. El tamaño observado en dichos gasterópodos es intermedio para conchas turriculadas (del orden de $5 \mathrm{~cm}$ de largo por $1 \mathrm{~cm}$ de ancho; las mayores conchas turriculadas corresponderían a algunas especies eocénicas del género Campanile), de paredes relativamente gruesas (aunque no pertenezca a estos ambientes, se puede poner como ejemplo de concha turriculada pequeña, la de Turritella spp. aff. dixoni Deshayes -ver 


\begin{tabular}{|c|c|c|c|c|c|c|}
\hline BIVALVOS-ESPECIES & \multicolumn{6}{|c|}{ DISTRIBUCION ESTRATIGRAFICA } \\
\hline \multirow{7}{*}{$\begin{array}{l}\text { Nuculana (Jupiteria) ilerdensis De Renzi } \\
\text { Barbatia (Rostarca) aff. exornata (Deshayes) } \\
\text { Dimya intusstriata (D'Archiac) } \\
\text { Gryphaeostrea eversa (Melleville) } \\
\text { Crassostrea sicardi (Doncieux) } \\
\text { - (Cubitostrea) multicostata (Deshayes) }\end{array}$} & cucu & clli & mouss & corbar & tremp & cuis \\
\hline & & PM,A & $? \mathrm{~A}$ & & & \\
\hline & PM & & & & & \\
\hline & & $\mathrm{A}$ & $? \mathrm{~A}$ & & & \\
\hline & & $\mathrm{T}, \mathrm{A}$ & $? \mathrm{~T}, ? \mathrm{~A}$ & & & \\
\hline & & & & & & PM \\
\hline & & & & $P$ & & \begin{tabular}{|l}
$\mathrm{M}, \mathrm{P}$ \\
$\mathrm{PM}$ \\
$\mathrm{AG}$ \\
\end{tabular} \\
\hline \multirow{5}{*}{$\begin{array}{l}\text { Pycnodonte sp. } \\
\text { Arcoperna profunda (Deshayes) } \\
\text { Septifer serratus (Melleville) } \\
\text { Botula cordata (Lamarck) } \\
\text { Bicorbula vidali (Cossmann) }\end{array}$} & & & & & & $\mathrm{AG}$ \\
\hline & & $\mathrm{A}$ & $? \mathrm{~A}$ & & & \\
\hline & & E & & & & \\
\hline & & & & & & $\mathrm{AG}$ \\
\hline & & & & & & \begin{tabular}{|c|}
$\mathrm{P}$ \\
$\mathrm{AG}$
\end{tabular} \\
\hline \multirow{6}{*}{\begin{tabular}{||l} 
Caryocorbula pixidicula (Deshayes) \\
Corbicula (s.s.) gravesi (Deshayes) \\
Callista (?) cf. o'gormani Cossmann \\
Venerella aff. cytheraeformis (Deshayes) \\
Katelysia (Textivenus) lignoni (Doncieux) \\
Megaximus (Codalucina)? cf. tuncinatus \\
$\quad$ (Defrance)
\end{tabular}} & & $\mathrm{A}$ & 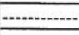 & $\mathrm{P}$ & & \\
\hline & & & & & & $\mathrm{AG}$ \\
\hline & & & & & & $\mathrm{AG}$ \\
\hline & & & & & & $\mathrm{AG}$ \\
\hline & $E$ & & & & & \\
\hline & E & & & & & \\
\hline \multirow{9}{*}{$\begin{array}{l}\text { Pseudomiltha (?) corbarica (Leymerie) } \\
\text { Cavilucina (?) cf. difficilis (Deshayes) } \\
\text { Psammodonax (?) cf. vaudini (Deshayes) } \\
\text { Venericardia submimuta (D'Orbigny) } \\
-\quad \text { junctinoda Cossmann } \\
\text { Goossensia insculpta (Deshayes) } \\
\text { Crassatella minima Leymeric } \\
-\quad \text { senessei Doncieux } \\
\text { Crassatella aff. securis Leymerie }\end{array}$} & $\begin{array}{c}\mathrm{P}, \mathrm{PC}, \mathrm{E}, \mathrm{T} \\
\mathrm{G}, \mathrm{A} \\
\end{array}$ & $\mathrm{E}, ? \mathrm{~T}, \mathrm{~A}$ & & & & \\
\hline & $\mathrm{PC}$ & & & & & \\
\hline & PM & & & & & \\
\hline & PM & $\mathrm{PM}, \mathrm{E}, \mathrm{A}$ & $? \mathrm{~A}$ & & & \\
\hline & & & & & & $\mathrm{AG}$ \\
\hline & & PM & & & & \\
\hline & & $\mathrm{A}$ & $? \mathrm{~A}$ & & & \\
\hline & $\mathrm{PM}$ & & & & & \\
\hline & & $E$ & & & & \\
\hline
\end{tabular}

Tabla 2. Listado de las especies de bivalvos marinos de la Conca de Tremp y La Vall d'Ager, con su distribución estratigráfica y por yacimientos, según los datos y orden taxonómico de De Renzi (1971). Siglas como en tabla 1.

más abajo-, con unos $3 \mathrm{~cm}$ de largo y 0.5 de ancho). Ello podría ser indicativo de que aunque este ambiente fuera de aguas salobres, tuviera una relativa estabilidad, oxígeno -esencial para el metabolismo y el crecimiento animal- y recursos, permitiendo a los organismos alcanzar un cierto tamaño (no obstante esto, ver más abajo); pero de acuerdo con los datos de Plaziat (1989, 1993 y 1995) podría ser también un medio marino litoral protegido.

Hay también abundantes moluscos en la parte alta de las secciones de la formación marina, y otra vez en medios parálicos, aunque con menor oscilación del mar (ello no es así en las secciones de la Vall d'Àger, en que la influencia marina es parecida a la observada en la Conca de Tremp al comienzo de la transgresión). Hay unos últimos niveles con alveolínidos y, por encima de ellos, aparecen materiales lutíticos con moluscos salobres en casi todos los cortes estudiados, a excepción de aquellos en que los conglomerados descansan en contacto ero- sivo sobre los materiales marinos y afloran únicamente las partes inferiores y medias de las secciones. En la sección por Areny tampoco han sido observados. Los moluscos salobres de estos niveles son: Potamides (Exechestoma) suturatus Cossmann, Pirenella (Tiaracerithium) figarolense (Doncieux), Tympanotonos (Eotympanotonus) turris orengae (Vidal in Cossmann), T. (Diptychochilus) montsecanus (Vidal in Cossmann), Tylochilus multigranosus (Doncieux), Pyrazus (s.s.) crusafonti De Renzi, etc. Pirenella (Tiaracerithium) figarolen$s e$ presenta unas enormes densidades que hacen pensar en un típico estratega $r$, aunque no parece que todas las formas asociadas a medios parálicos tengan por qué serlo; así, los Melanopsis actuales que he tenido ocasión de observar en el marjal de Torreblanca (Castellón), no exhiben grandes densidades.

El otro conjunto importante son las margas de Turritella, con tres especies: T. dixoni Deshayes -en De Renzi (1971) está justificado el motivo por el cual $T$. trempina Carez es sinónima de aquélla-, $T$. figolina Carez y $T$. spp. aff. dixoni Deshayes. Estos turritélidos suelen estar diseminados en el sedimento (ver TAFONOMIA), sin llegar, por lo general, a grandes densidades, aunque son muy abundantes en los productos de meteorización. Su situación en la cuenca sería ya bastante distal (Luterbacher, 1970). T. figolina quizá sería la forma relacionada con condiciones más marinas; se asocia con corales solitarios, dados inicialmente como "Pattalophyllia", que actualmente estudia el Dr. Germán Alvarez. T. dixoni y $T$. figolina van acompañadas, normalmente, por abundantes Operculina. En una ocasión he extraído en dichos niveles algún gran fragmento vegetal, lo cual parece de acuerdo con el carácter poco profundo y relativamente proximal en que fueron depositados, y que parece inferirse por otras evidencias. Muy probablemente se podría considerar ambas especies como estrategas $K$ en un ambiente estable (los grandes foraminíferos del género Operculina, como en general los macroforaminíferos, son típicos estrategas de $K$; Hottinger, 1984). Sin embargo, T. spp. aff. dixoni es una forma de pequeña talla, con grandes densidades, en materiales sin macroforaminíferos. Su ornamentación parece detenerse en fases que parecen corresponderse con las de los estadios juveniles de $T$. dixoni y con menor tasa de expansión de la vuelta que ésta, lo cual le da apariencia acicular. Ambas características, tamaño y ornamentación, apuntarían a una forma progenética, pero no necesariamente derivada de $T$. dixoni, sino de alguna forma próxima a aquella; ello, junto con la densidad relativamente alta, parece caracterizarla como un estratega $r$ (Gould, 1977). La carencia de macroforaminíferos podría

\begin{tabular}{|c|c|c|c|c|c|}
\hline SEDIMENTIVOROS & $\begin{array}{c}\text { EPIBIONTES } \\
\text { SUSPENSIVOROS }\end{array}$ & $\begin{array}{l}\text { ENDOBIONTES } \\
\text { NO SIFONADOS }\end{array}$ & ENDOBIONTES & ENDOBIONTES & ATTRIBUCION \\
\hline Nucu 7 ana & $\begin{array}{l}\text { Dimya } \\
\text { Gryphaeostrea } \\
\text { Crassostrea } \\
\text { Pycnodonte }\end{array}$ & Crassate77a & $\begin{array}{l}\text { Bicorbula } \\
\text { Caryocorbula } \\
\text { Gouldia } \\
\text { Cal7ista } \\
\text { Venerel7a } \\
\text { Katelysia } \\
\text { Psammodonax }\end{array}$ & $\begin{array}{l}\text { Megaxinus } \\
\text { Pseudomi tha } \\
\text { Cavilucina }\end{array}$ & $\begin{array}{l}\text { Barbatia } \\
\text { Septifer } \\
\text { Arcoperna } \\
\text { Botula } \\
\text { Corbicula } \\
\text { Venericardia }\end{array}$ \\
\hline TOTALES: 1 & 4 & 1 & 7 & 3 & 6 \\
\hline
\end{tabular}

Tabla 3. Clasificación de los modos de vida de los géneros de bivalvos de la Conca de Tremp y La Vall d'Ager, de acuerdo con Stanley (1968). 
apoyar esta suposición; son especies que requieren un ambiente estable y su ausencia podría evidenciar un estrés en el medio, lo cual sería causa de que estuviera habitado por formas típicamente oportunistas, como la que nos ocupa.

Los modos de vida son interesantes. Los bivalvos, de acuerdo con Stanley (1968), poseen cinco tipos tróficos; la distribución de los 21 géneros que aquí mencionamos en dichos grupos viene dada en la Tabla 3. A excepción del único caso de endobiontes "labial palp deposit feeders", que se asocia únicamente a los depósitos margosos distales (posiblemente zonas tranquilas), y de los endobiontes de tubo mucoso (Lucinacea) que parecen relacionados con zonas intermareales o de energía en general (excavación profunda a causa de la erosión debida a corrientes), los otros endobiontes (los no sifonados y los sifonados) se hallan tanto en facies someras como distales. Los epibiontes suspensívoros también forman un amplio abánico en cuanto a habitats.

Los modos de vida de los gasterópodos no son algo tan bien establecido como ocurre en los bivalvos, puesto que en estos últimos las características de la concha están muy ligadas al sustrato, mientras que en los primeros ello no es así (Stanley, 1988). Signor (1982) ha contribuido a la comprensión del significado funcional de las conchas turriteliformes, y Savazzi (1989) ha hecho otro tanto en lo que se refiere a las relaciones entre tipo de ornamentación y excavación (burrowing) en gasterópodos en general; dichos trabajos pueden servir como fundamento de lo que voy a exponer a continuación.

Los gasterópodos turriteliformes (Signor, 1982) presentan modos de vida que pueden predecirse con cierta fiabilidad de las características de sus conchas. Pueden ser epibiontes móviles, sedentarios (epi y endobiontes) y excavadores activos. De acuerdo con la discusión de dicho autor-que empieza por afirmar que no está dando leyes sino generalizaciones empíricas, con las excepciones que puedan haber-, de nuestros turriteliformes podríamos citar Batillaria (Vicinocerithium) couizensis Doncieux o Tympanotonos (Eotympanotonus) turris orengae (Vidal in Cossmann) como formas epibiontes sedentarias o móviles. Ello a causa de sus fuertes tubérculos espinosos, como defensa contra la predación sobre el fondo. Los excavadores activos se suelen caracterizar por sus vueltas de espira de muy baja convexidad, lisas o con ornamentación aterrazada (ratchet sculpture); todo ello facilita el delizamiento a través del sedimento. Aquí los ejemplos serían Ptychocerithium multivariculosum (Cossmann), Bezançonia cossmanni (Oppenheim) y Bittium (?) aff. plicatulum (Deshayes). De entre las especies de la familia Turritellidae, Turritella dufrenoyi Leymerie sería la única en cumplir algunos de estos requisitos. En cambio, $T$. dixoni Deshayes, $T$. figolina Carez y otras menos frecuentes (ver Tabla 1), por sus carenas hacen pensar en la dificultad inherente a la excavación activa. Probablemente se trate de organismos sedentarios o epibiontes móviles. A favor de su carácter sedentario endobionte se podría citar la rareza de fenómenos tales como incrustación por epizoos.

\section{EL REGISTRO HORIZONTAL Y VERTICAL DE LOS MOLUSCOS}

Los factores tafonómicos y paleoecológiccos van a condicionar la distribución tanto horizontal como vertical de los moluscos en el Paleógeno marino de la Conca de Tremp. Una primera cosa que llama la atención es la enorme desproporción entre gasterópodos y bivalvos. Los primeros constituyen el $76 \%$, mientras que los segundos únicamente representan el $24 \%$ de los moluscos recogidos. Una explicación podría ser que el distinto comportamiento hidrodinámico que tienen unos y otros sea determinante a la hora de su registro (las conchas de bivalvos muertos son más fáciles de transportar que las más cónicas de los gasterópodos; Martinell, comunicación personal), aunque tampoco es muy segura, puesto que la mayor parte de los bivalvos hallados están cerrados.

Los moluscos aragoníticos no se hallan en los materiales más proximales, que se sitúan al S de la Conca (predominio de la sedimentación carbonática y detrítica más o menos grosera y organógena) por los condicionamientos tafonómicos ya mencionados anteriormente; ello no es obstáculo para que en algunos materiales margosos intercalados se halle, más de una vez, moluscos aragoníticos con su concha, pero no muy abundantes, en general deformados y, en muchos casos, indeterminables. La mayor preponderancia de los sedimentos lutíticos hacia el centro y el $\mathrm{N}$ de la Conca hace que éste sea, dentro de lo que es una conservación muy desigual, el dominio de este tipo de fósiles. Ni que decir tiene que las conchas calcíticas o biminerálicas son ubicuas en ambos tipos de materiales.

Las secciones muestran una transgresión progresiva a la que sucede, también con carácter progresivo, una regresión, que más o menos sería, desde el punto de vista de la sucesión de capas, como una imagen especular de la primera. Los contenidos paleontológicos, en lo referente a formas bentónicas, son correlativos con estos regímenes ecológicos. De este modo, los moluscos de ambientes parálicos (o marinos litorales protegidos), con las salvedades debidas a la conservación, se registran fundamentalmente en los episodios iniciales de la transgresión o en las fases finales de la regresión y, sobre todo, hacia el centro y el $\mathrm{N}$ de la Conca de Tremp, por todo lo dicho. Aunque la transgresión se deja sentir en toda la zona, el dominio de las distintas especies de Turritella se da en los depósitos más distales, constituídos por margas muy arcillosas.

Los depósitos de playa que preceden a los términos finales de la regresión suelen estar constituídos por lutitas que contienen proporciones variables de limo (cf. Luterbacher, 1970; p. 28 y fig. 32), y en las que se hallan Turritella muy mal conservadas y escasas. Este tipo de materiales han sido constatados inmediatamente por encima de Fígols de Tremp y del pueblo de Eroles.

\section{ENSAYO DE UNA BIOESTRATIGRAFÍA DE MOLUSCOS}

Las Tablas 1 y 2 mostraban la repartición de los moluscos de acuerdo con una de las biozonaciones -la de los alveolínidos- aceptada al uso (Molina et al., 1992). Esto se ha efectuado tomando como referencia la sección tipo (carretera de Tremp a Puente de Montañana) que publican los mismos autores, con los cuerpos de roca que abarca cada biozona. Otros trabajos (De Renzi, 1968; Ferrer et al., 1973) han permitido colocar los moluscos en las secciones de Areny y de Tendruy-Sant Adrià de acuerdo con dicha biozonación. El trabajo de Hottinger (1960) permitió, asimismo, la datación 


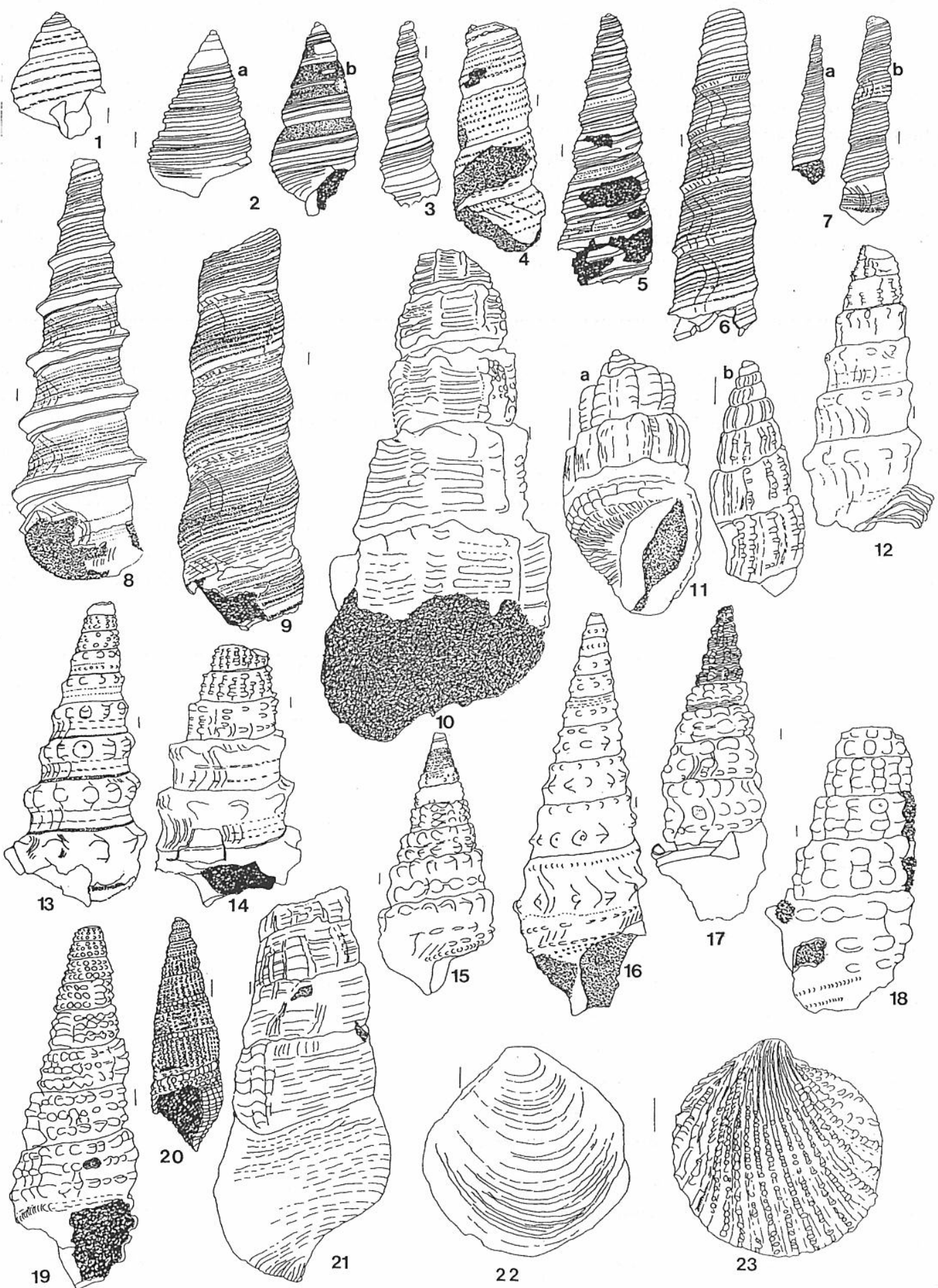

Figura 3. Especies de moluscos con interés estratigráfico para el área tipo; las siglas dan el nombre del corte y de la biozona donde se halló el especimen figurado: T, Tremp-Puente de Montañana; E. Eroles; A. Areny; TS, Tendruy-Sant Adrià; PGC, Puigcercós; cuc, Cucumiformis; elli, Ellipsoidalis; mou, Moussoulensis. 1. Homalopoma (s.s.) conoideum; (E, elli). 2a, b, Sigmesalia spp.; (A, elli). 3, Zaria (?) vaudini; (T, cuc). 4, Z. (?) quinquecarinata; (E, elli). 5, Z. (?) caroli; (TS, ? mous). 6, Turritella dixoni; (T, elli). 7 a, b, T. spp. aff. dixoni; (A, elli). 8, T. figolina; (T, mous). 9, T. dufrenoyi; (A, elli). 10, Melanatria almerae; (T, cuc). 11 a, b, Melanopsis (Canthidomus) vidali; (T, cuc). 12, Pirenella (Tiaracerithium) almerae; (TS, cuc). 13, Tympanotonos (Eotympanotonus) hypermeces; (TS, cuc). 14, T. (E.) turris orengae; (T, cuc). 15, T. (E.) inaequirugatus; (T, cuc). 16, Batillaria (Vicinocerithium) couizensis; (T, cuc). 17, B. (V.) praesubacuta; (T, cuc). 18, B. (V.) inopinata puigcercosensis; (T, cuc). 19, Bittium (?) richei; (E, cuc). 20, Fastigiella (Mellevillia) gibbosula (T, cuc). 21, Ptychocerithium multivariculosum; (T, cuc). 22, Pseudomiltha (?) corbarica; (PGC, cuc). 23, Venericardia subminuta; (T, elli). Dibujos a cámara clara por el autor, con ejemplares de su colección. Longitud de la barra $1 \mathrm{~mm}$ para todos los ejemplares, menos para el 22 , que equivale a $1 \mathrm{~cm}$. 
de los cortes por Puigmaçana y Mur. Estos últimos se prestaban a una cierta vaguedad en cuanto a la delimitación de las biozonas, lo cual dio origen a algunas afirmaciones erróneas acerca del límite Ilerdiense inferior-Ilerdiense medio en De Renzi $(1967,1971)$ que aquí se rectifican. El corte por Eroles se puede interpolar bien entre el de Tendruy y el de la sección tipo. No obstante, en este corte tengo dudas acerca del límite entre la Biozona Cucumiformis y la Biozona Ellipsoidalis, cosa que se está estudiando en este momento. Por tanto, tres de los marcadores que en esta sección entran en la supuesta parte inferior de la Biozona Ellipsoidalis, podrían llegar únicamente a la parte superior de la Biozona Cucumiformis (ver tabla 4), y lo mismo para otros moluscos señalados en este corte, por debajo de las lutitas con Turritella (Tablas 1 y 3). Por otra parte, en De Renzi $(1968,1971)$ y Crusafont, Rosell et al. (1968) se justificaba que las partes más altas de las secciones marinas de la Formación Ager en la región estudiada (que comprendería el área tipo del Ilerdiense) había que datarlas como de edad Cuisiense inferior.

Un total de 23 especies ( 21 gasterópodos y 2 bivalvos) pueden constituir formalmente un conjunto de marcadores (Tabla 4 y Fig. 3). Su elección se ha basado en que bastantes de ellos aparecen, por vez primera, en más de un corte y en la misma biozona; además, existe una notable homotaxia en cuanto a la aparición sucesiva de muchos de estos fósiles, mientras que otros se restringen a un intervalo bastante estrecho de tiempo. Únicamente en el caso de Turritella spp. aff. dixoni Deshayes y $T$. dufrenoyi Leymerie, existen problemas. Sus primeras apariciones presentan una regularidad notable en varios cortes: primero aparece $T$. dufrenoyi, después Turritella spp. aff. dixoni y, por último, T. dixoni Deshayes. Pues bien, en el corte de Tendruy las primeras apariciones de ambas se dan después de la primera aparición de $T$. dixoni y de Sigmesalia spp. Ha sido la homotaxia entre los distintos cortes la que ha permitido usar la primera aparición de Turritella figolina como un buen marcador del límite entre las Biozonas Ellipsoidalis y Moussoulensis.

La ausencia general de condiciones de conservación para los moluscos aragoníticos a partir de la parte media de los materiales de la Biozona Moussoulensis hasta parte de los de la Biozona Trempina no permite dar otros marcadores que hubieran aparecido de poderse conservar.

Ahora bien, los organismos bentónicos, en una situación de transgresión-regresión como la que se acaba de describir, presentan unas enormes dificultades en cuanto a un posible uso bioestratigráfico. Unicamente un conocimiento tafonó-

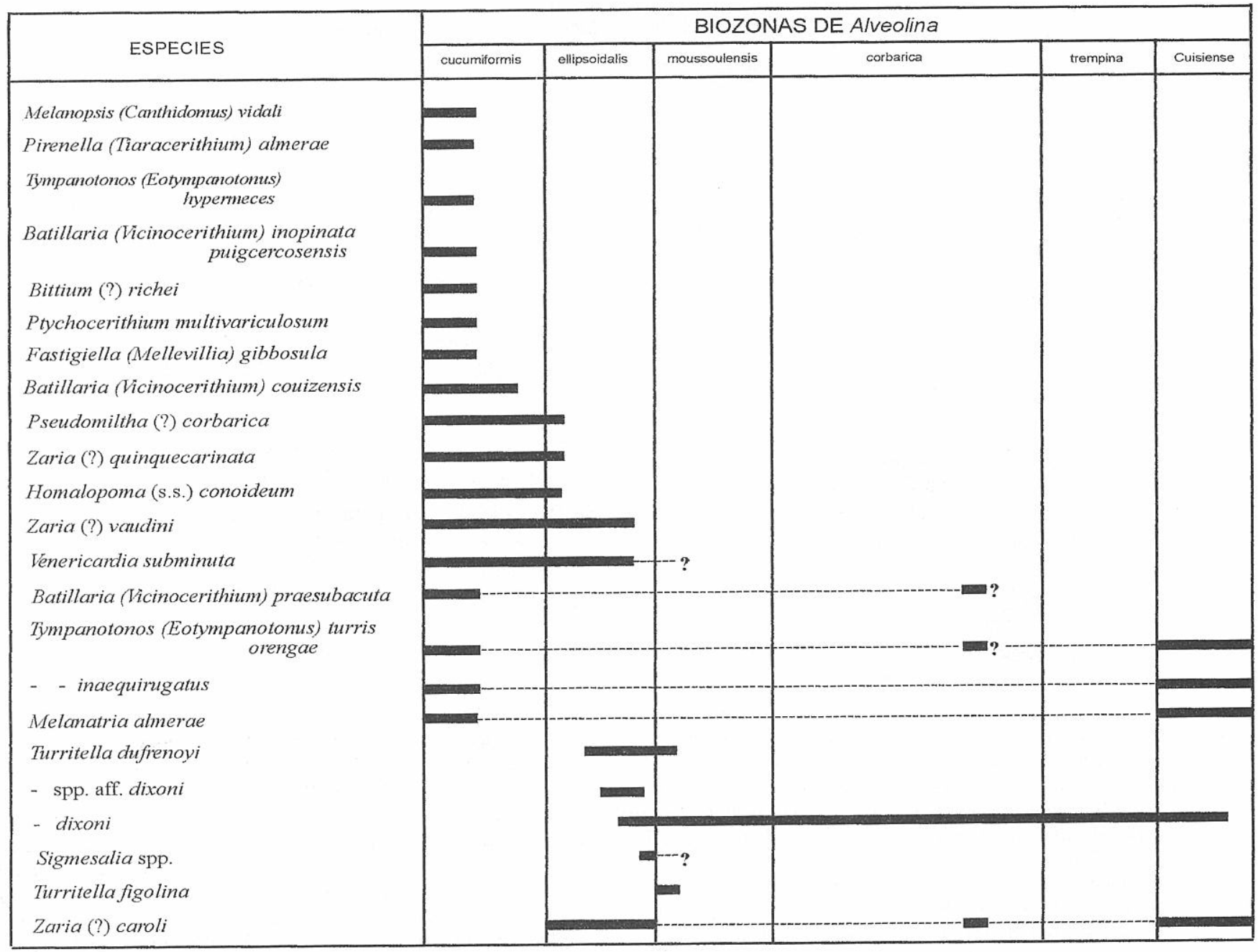

Tabla 4. Propuesta de 23 marcadores (con valor únicamente local) para el área tipo del Ilerdiense (ver texto). 
mico y paleoecológico previo puede permitir obtener alguna conclusión al respecto. La biozonación mediante alveolínidos (Hottinger, 1960) aquí utilizada presentaría, pues, problemas. Su autor muestra, paradójicamente, cómo las características morfológicas de las Alveolina están más o menos asociadas a facies (pp. 22-24). Así, según él, no es nada raro que el grupo de Alveolina ellipsoidalis, base de la escala bioestratigráfica del Ilerdiense y que constituye un "rameau phylétique" del género Alveolina, posea una "continuité exceptionelle $[. .$.$] due au fait que le faciès, où les formes de ce$ groupe se montrent de préférence, devient plus détritique avec l'allongement de plus en plus accusé des espèces" (p. 63). Ello quiere decir que formas de distinto alargamiento pueden ser perfectamente isócronas, ya que las facies que las contienen pueden serlo perfectamente. Lo mismo cabría decir para el aplanamiento progresivo de los nummulítidos que se observa al ascender por las distintas secciones estratigráficas. Alargamiento y aplanamiento pueden ser, en parte, respuestas a las necesidades de luz de los simbiontes fotosintéticos residentes en el citoplasma de los macroforaminíferos. Turbidez y/o profundidad determinarán la necesidad de una mayor superficie foto-receptora (Leutenegger, 1984; De Renzi, 1988b). Por tanto, las biozonaciones propuestas pueden correlacionar más bien ambientes que momentos del tiempo geológico.

Algo parecido puede decirse acerca de las primeras apariciones de los moluscos que aquí se consideran. Los cuerpos rocosos atribuibles a la Biozona Cucumiformis suelen corresponder a sedimentos depositados en ambientes parálicos a marinos muy someros, mientras que los de las Biozonas Ellipsoidalis y Moussoulensis representarían un gradiente progresivo de oceanicidad y, algunas veces, profundidad. Se puede decir que únicamente las primeras apariciones en las margas de Turritella pudieran tener un valor al respecto, ya que no dependerían tanto de factores ecológicos como evolutivos y de capacidad de dispersión de los organismos pretéritos implicados.

\section{CONCLUSIONES}

Lo dicho muestra cómo cualquier intento de bioestratigrafía ha de considerar previamente los aspectos tafonómicos y paleobiológicos del grupo que se va usar con tal finalidad. El valor de los moluscos usados con este fin para la bioestratigrafía del estratotipo del Ilerdiense tiene carácter local y, además, no abarca el área tipo. Muchas de las especies aquí halladas se las encuentra a partir del Cuisiense por primera vez en otras cuencas (De Renzi, 1971, 1972); por ejemplo, en Gan y en la cuenca de París, con todas las salvedades que pueda originar el término Cuisiense. Incluso hay formas típicamente lutecienses. Sin embargo, de los 23 marcadores propuestos, algunos de ellos serían muy característicos de la Biozona Cucumiformis: Melanopsis (Canthidomus) vidali, Pirenella (Tiaracerithium) almerae y Tympanotonos (Eotympanotonus) hypermeces. Turritella spp. aff. dixoni lo sería de la Biozona Ellipsoidalis, mientras que $T$. figolina caracterizaría la base de la Biozona Moussoulensis.

La zonación propuesta, a pesar de su carácter local, permite caracterizar bien el Ilerdiense inferior y la base del Ilerdiense medio.

\section{AGRADECIMIENTOS}

Este trabajo resume diversos aspectos dados en De Renzi (1971) y que no fueron publicados por diversos motivos; doy las gracias a los que fueron mis maestros entonces: los Doctores Miquel Crusafont y Maxime Glibert. Referido a este trabajo, agradezco su lectura y comentarios al Dr. Eustoquio Molina (Depto. de Geología; Paleontología; Universidad de Zaragoza). Al mismo tiempo, hay nuevos modos de ver las cosas y nuevos datos; todo ello ha sido desarrollado fundamentalmente a partir del Proyecto de la CAICYT 2934/83.C2 y el Proyecto de la DGICYT PB91-0644. Por último, doy las gracias al señor Miguel Vicente Pardo (Dept. de Geologia, Universitat de València) por su ayuda para mejorar el gráfico de las columnas mediante scanner.

\section{BIBLIOGRAFÍA}

Barnolas, A., Robador, A., Serra-Kiel, J. and Caus, E. (Eds.) 1990. Introduction to the Early Paleogene of the South Pyrenean Basin. Field-Trip Guidebook. 159 pp. Early Paleogene Benthos First Meeting. Jaca, Spain.

Carez, L. 1881. Étude des terrains crétacés et tertiaires du Nord de l'Espagne. Librairie F. Savy. París. 323 pp.

Cossmann, M. 1897. Estudio de algunos moluscos eocenos del Pirineo catalán. Boletín de la Comisión del Mapa Geológico de España, 23, 167-198.

Cossmann, M. 1906. Estudio de algunos moluscos eocénicos del Pirineo catalán. Boletín de la Comisión del Mapa Geológico de España, 28, 135-151.

Crusafont, M., De Renzi, M. et Clavell, E. 1968. Les grands traits d'une coupure crétacé-paléocène-éocène au Sud des Pyrénées (Isábena). Mémoires du Bureau de Recherches Géologiques et Minières, 58 (Colloque sur l'Éocène), 591-596.

Crusafont, M., Rosell, J., Golpe, J. M. et De Renzi, M. 1968. Le Paléogène de la vallée d'Ager et ses rapports avec celui de la Conca de Tremp (Pyrénées de la province de Lérida, Espagne). Mémoires du Bureau de Recherches Géologiques et Minières, 58 (Colloque sur l'Éocène), 584-589.

Dalloni, M. 1930. Étude géologique des Pyrénées catalanes. Annales de la Faculté des Sciences de Marseille, 26, 1-373.

De Renzi, M. 1966. El problema del límite entre Secundario y Terciario en las proximidades de Serraduy, en el valle del Isábena (provincia de Huesca). Acta Geológica Hispánica, 2, 3-8.

De Renzi, M. 1967. Nota sobre la estratigrafía del Paleoceno superior marino de la Conca de Tremp, basada en las faunas de moluscos. Revista del Instituto de Investigaciones Geológicas de la Diputación Provincial, 21, 39-50.

De Renzi, M. 1968. Trois nouvelles coupes de l'Ilerdien marin de l'Aragon et leurs rapports avec le stratotype de l'Ilerdien à la Conca de Tremp (Espagne). Mémoires du Bureau de Recherches Géologiques et Minières, 58 (Colloque sur l'Éocène), 597-606.

De Renzi, M. 1971. Las faunas de moluscos fósiles del Eoceno inferior del Prepirineo de Lérida. Tesis doctoral inédita, Universidad de Barcelona. 502 pp.

De Renzi, M. 1972. Las faunas de moluscos fósiles del Eoceno inferior del Prepirineo de Lérida. Resumen de la Tesis presentada para aspirar al grado de Doctor en Ciencias. Universidad 
de Barcelona. Secretariado de Publicaciones, Intercambio Científico y Extensión Universitaria. 10 pp.

De Renzi, M. 1975. Sur la répartition des mollusques dans le stratotype de l'Ilerdien en rapport avec les faunes de Mollusques de l'Éocène européen. Bulletin de la Société géologique de France, 17, 199-200.

De Renzi, M. 1978. La problemática de la fundamentación de la Paleoecología. Estudios geológicos, 34, 559-570.

De Renzi, M. 1981. Some philosophical questions about paleontology and their practical consequences. Concept and method in Paleontology. Invited Papers (Ed. J. Martinell). Acta Geológica Hispánica, 16, 7-23.

De Renzi, M. 1988a. Actualismo, uniformismo y paleontología: hacia un mejor entendimiento entre paleontólogos y sedimentólogos. II Congreso Geológico de España, Comunicaciones, I, 228-232.

De Renzi, M. 1988b. Shell coiling in some larger foraminifera: general comments and problems. Paleobiology, 14, 387-400.

Doncieux, L. 1903. Monographie géologique et paléontologique des Corbières orientales. Annales de l'Université de Lyon. Nouvelle Série. I. Sciences, Médecine, 11, 1-377.

Doncieux, L. 1905. Catalogue descriptif des fossiles nummulitiques de l'Aude et de l'Hérault. Première partie. Montagne Noire et Minervois. Annales de l'Université de Lyon. Nouvelle Série. I. Sciences, Médecine, 17, 5-164.

Doncieux, L. 1908. Catalogue descriptif des fossiles nummulitiques de l'Aude et de l'Hérault. Deuxième partie (Fascicule I). Corbières septentrionales. Annales de l'Université de Lyon. Nouvelle Série. I. Sciences, Médecine, 22, 1-250.

Doncieux, L. 1911. Catalogue descriptif des fossiles nummulitiques de l'Aude et de l'Hérault. Deuxième partie (Fascicule II). Corbières septentrionales. Annales de l'Université de Lyon. Nouvelle Série. I. Sciences, Médecine, 30, 1-199.

Ferrer, J., Le Calvez, Y., Luterbacher, H. et Premoli Silva, I. 1973. Contribution à l'étude des foraminifères ilerdiens de la région de Tremp (Catalogne). Mémoires du Muséum National d'Histoire Naturelle. Nouvelle Série, Série C, Sciences de la Terre, 29, 1-107.

Fischer, P. 1887. Manuel de Conchyliologie et de Paléontologie conchyliologique ou Histoire Naturelle des Mollusques vivants et fossiles. Librairie F. Savy. París. 1369 pp.

Gould, S.J. 1977. Ontogeny and phylogeny. The Belknap Press of Harvard University Press. Cambridge, Mass. \& London, England. $501 \mathrm{pp}$.

Hottinger, L. 1960. Recherches sur les Alvéolines paléocènes et éocènes. Mémoires Suisses de Paléontologie, 75, 1-243.

Hottinger, L. 1984. Foraminifères de grand taille: signification des structures complexes de la coquille. Benthos'83; $2^{\text {nd }}$ International Symposium on Benthic Foraminifera, 309-315.

Hottinger, L. y Schaub, H. 1960. División en pisos del Paleoceno y del Eoceno. (Trad. esp. J.M. Rios, 1961). Notas y Comunicaciones, 61, 199-233.

Luterbacher, H. 1970. Environmental distribution of Early Tertiary microfossils, Tremp Basin, Northeastern Spain. Esso Production Research-European Laboratories. 48 pp.
Luterbacher, H. 1973. La sección tipo del piso Ilerdiense. XIII Coloquio Europeo de Micropaleontología (no especifica editor), 113-140.

Leutenegger, S. 1984. Symbiosis in benthic foraminifera: specificity and host adaptations. Journal of Foraminiferal Research, 14, 16-35.

Llompart, C. 1977. Paleoecología de la fauna de moluscos ilerdienses en un sector de La Vall d'Ager (Provincia de Lleida). Publicaciones de Geología, 7, 251 pp. Universidad Autónoma de Barcelona.

Molina, E., Canudo, J. I., Guernet, C., McDougall, K., Ortiz, N., Pascual, J. O., Parés, J. M., Samsó, J. M., Serra-Kiel, J. and Tosquella, J. 1992. The stratotypic Ilerdian revisited: integrated stratigraphy across the Paleocene/Eocene boundary. Revue de Micropaléontologie, 35(2), 143-156.

Mutti, E., Luterbacher, H. P., Ferrer, J. e Rosell, J. 1972. Schema stratigrafico e lineamenti di facies del Paleogene marino della zona centrale sudpirenaica tra Tremp (Catalogna) e Pamplona (Navarra). Memorie della Societá Geologica Italiana, 11, 391-416.

Plaziat, J.-C. 1989. Signification écologique et paléogéographique des peuplements oligotypiques de potamides gastéropodes thalassiques. Atti $3 .^{\circ}$ Simposio di Ecologia e Paleoecologia delle Comunità Bentoniche, a cura di S. Italo di Geronimo; Catania-Taormina, 1985, 25-52.

Plaziat, J.-C. 1993. Modern and fossil Potamids (Gastropoda) in saline lakes. Journal of Paleolimnology, 8, 163-169.

Plaziat, J.-C. 1995. Modern and fossil mangroves and mangals: their climatic and biogeographic variability. In: Marine Palaeoenvironmental Analysis from Fossils (Eds. D.W.J. Bosence \& P.A. Allison), Geological Society Special Publication, 83, 73-96.

Plaziat, J.-C. et De Renzi, M. 1968. Corrélation, à l'aide des macrofaunes marines, entre l'Ilerdien du bassin de Tremp (Lérida, Espagne) et la série cuiso-lutétienne des Corbières (Aude, France). Mémoires du Bureau de Recherches Géologiques et Minières, 58 (Colloque sur l'Éocène), 575-581.

Savazzi, E. 1989. Burrowing mechanisms and sculptures in Recent gastropods. Lethaia, 22, 31-48.

Serra-Kiel, J., Canudo, J. I., Dinarès, J., Molina, E., Ortiz, N., Pascual, J. O., Samsó, J. M. y Tosquella, J. 1994. Cronoestratigrafía de los sedimentos marinos del Terciario inferior de la Cuenca de Graus-Tremp (Zona Central Surpirenaica). Revista de la Sociedad Geológica de España, 7(3-4), 273-297.

Signor, P. W., III. 1982. Resolution of life habits using multiple morphologic criteria: shell form and life-mode in turritelliform gastropods. Paleobiology, 8, 378-388.

Stanley, S. M. 1968. Post-Paleozoic adaptive radiation of infaunal bivalve molluscs-A consequence of mantle fusion and siphon formation. Journal of Paleontology, 42(1), 214-229.

Stanley, S. M. 1988. Adaptive morphology of the shell in bivalves and gastropods. In: The Mollusca. Volume 11. Form and function (Eds. E. R. Trueman and M. R. Clarke). Academic Press, Inc. Harcourt Brace Jovanovich, Publishers. San Diego, New York, London. 105-141.

Manuscrito recibido: 10 de enero, 1995 Manuscrito aceptado: 4 de julio, 1995 